\title{
Biosimilars- An opportunity to update the Product Information of biological drugs regarding their immunogenicity
}

\author{
Rodrigo Borrega ${ }^{1}$ (ORCID ID 0000-0001-7627-7223), Joao Paulo Cruz ${ }^{1,2}$, Peter Taylor³, \\ Joao Goncalves ${ }^{1}$ (ORCID ID $\underline{\text { 0000-0002-1245-3715) }}$ \\ 1 iMed.ULisboa-Research Institute for Medicines, Faculty of Pharmacy, Universidade \\ de Lisboa, Lisbon, Portugal \\ 2 Pharmacy Service, Centro Hospitalar Lisboa Norte/Hospital de Santa Maria, Lisbon, \\ Portugal
}

${ }^{3}$ Nuffield Department of Orthopaedics, Rheumatology and Musculoskeletal Sciences, Kennedy Institute of Rheumatology, University of Oxford, Botnar Research Centre, Oxford, UK

Correspondence: joao.goncalves@ff.ul.pt (+351 217946486)

Running Heading: Biosimilars as an opportunity to update the Product Information of biological drugs

One of the greatest theoretical clinical concerns regarding the development of biosimilars has been that post-translational protein modifications, even while antigen binding remains essentially unaltered, might result in detrimental immunogenicity. We recently demonstrated [1] that the Summary of Product Characteristics (SmPCs) of biological drugs, approved by the European Medicines Agency (EMA), are very heterogeneous regarding the issues related to immunogenicity that are addressed within these documents. A complementary analysis, using the methodology advanced in [1], to the EMA's documents "Procedural steps taken and scientific information after the authorisation" shows that $57 \%$ (30/69) of the biological drugs that have been authorized prior to 2012 did not update their SmPC at least once when it comes to information related to unwanted immunogenicity. These are surprising results given the advancements that have been seen in the field of immunogenicity assessment [2], namely in the development of more 
sensitive and drug tolerant assays. These recent assays detect Anti-Drug Antibodies (ADAs) in a significantly greater proportion of patients and are being commonly employed in Bioequivalence studies in order to detect, if present, small differences between Biosimilars and the Reference Product. On the other hand, immunogenicity results reported in the SmPCs of these Reference Products are usually based on assays developed and validated 10-15 years ago. Given that the immunogenicity rates reported by SmPCs influence the perception of the medical community about how often the development of an immunogenic response against a biological drug occurs, we wonder if the SmPCs of older drugs should not be updated, when data is available, to also reflect the immunogenicity rates that are being detected with these newer assays. Thus, a perfect opportunity to update the information in some of these SmPCs arises from the approval of biosimilars.

According to European guidelines, a biosimilar is defined as a biological medicine that is a highly similar version of an already established biological drug (reference product) in the European Economic Area. Therefore, getting approval into the European Market requires that these products demonstrate a high degree of similarity (in terms of quality, biological and medical properties) between themselves and their counterparts [3]. This assessment includes head to head clinical studies that compare a biosimilar's immunogenicity profile to its' Reference Product and this analysis is extremely relevant due to the low predictability that non-clinical models confer regarding the unwanted immunogenicity of biological drugs.

The Biosimilar Medicinal Products Working Party (BMWP) reported in 2012 three different options for reporting of bioequivalence data in the SmPCs of biosimilar drugs. These options ranged from not including any information at all up to only reporting the bioequivalence data supporting EMA's assessment [4]. The decision that came from this discussion was that a Marketing Authorisation Holder must develop a document identical (with the exception of using the International Nonproprietary Name of the active substance instead of the tradename of the reference product [5]) to the SmPC of the reference product, thus excluding any bioequivalence data from the SmPCs and include it exclusively in the product's European Public Assessment Report (EPAR). We argue that this is an inadequate strategy given the low proportions of Healthcare Professionals that use EPARs as a source of information [6]. Additionally, we would argue that excluding information about the bioequivalence studies in the SmPCs increases the 
likelihood of misunderstandings and unreasonable fears by some members of the medical community. On the other hand, the regulators' stance that different SmPCs between products with a similar active substance could lead to misunderstandings is also sensible [7]. Therefore, considering both sides of the argument, we propose the idea that both the SmPCs of the reference product and of the biosimilar product should include data from the bioequivalence studies, specifically the data related to the immunogenicity.

From the reference products' perspective, we argue that this update is necessary given that most of these drugs have been on the market for over 10 years. During this period, an increasingly higher number of improvements regarding the assessment of immunogenicity have occurred but many SmPCs include data mostly obtained during the drug's pivotal clinical trials [8], thus possibly underestimating the immunogenic potential of some products [9]. Consequently, the inclusion of immunogenicity data from bioequivalence studies, assessed with new methodologies, could be an excellent opportunity to update these drugs' SmPCs in order to provide a better sense of amplitude about the detected incidence of ADAs.

While the possibility of including information in the SmPCs of both the Reference Product and of the biosimilar drugs had not been considered by the BMWP, this is not a novel solution. Common updates, regarding immunogenicity, to SmPCs of different biological products that contain the same or closely related active substance can be seen in several situations identified in Table 1. Examples of these include the updates A31/0134 and A31/0178 for moroctocog alfa (ReFacto AF) and octocog alfa (Helixate NexGen) respectively $[13,14]$ or the update IB/0002/G for epoetin theta (Biopoin and Eporatio) $[15,16]$.

From the biosimilars' perspective, we argue that this update is necessary because issues such as immunogenicity [10], switching/interchangeability [11, 12] and extrapolation of indications [7] have been raised throughout the years. Inclusion of immunogenicity data collected during the bioequivalence studies can help clarify some of these issues in a document that is regularly used by HCPs. Additionally, by reporting data that supports the decision to approve a biosimilar into the European Market might help invalidate the perception that the evidence supporting this decision is insufficient [7], while excluding data from bioequivalence studies foments this perception. 
Table 1- Common updates to the immunogenicity information addressed by SmPCs of different biological products

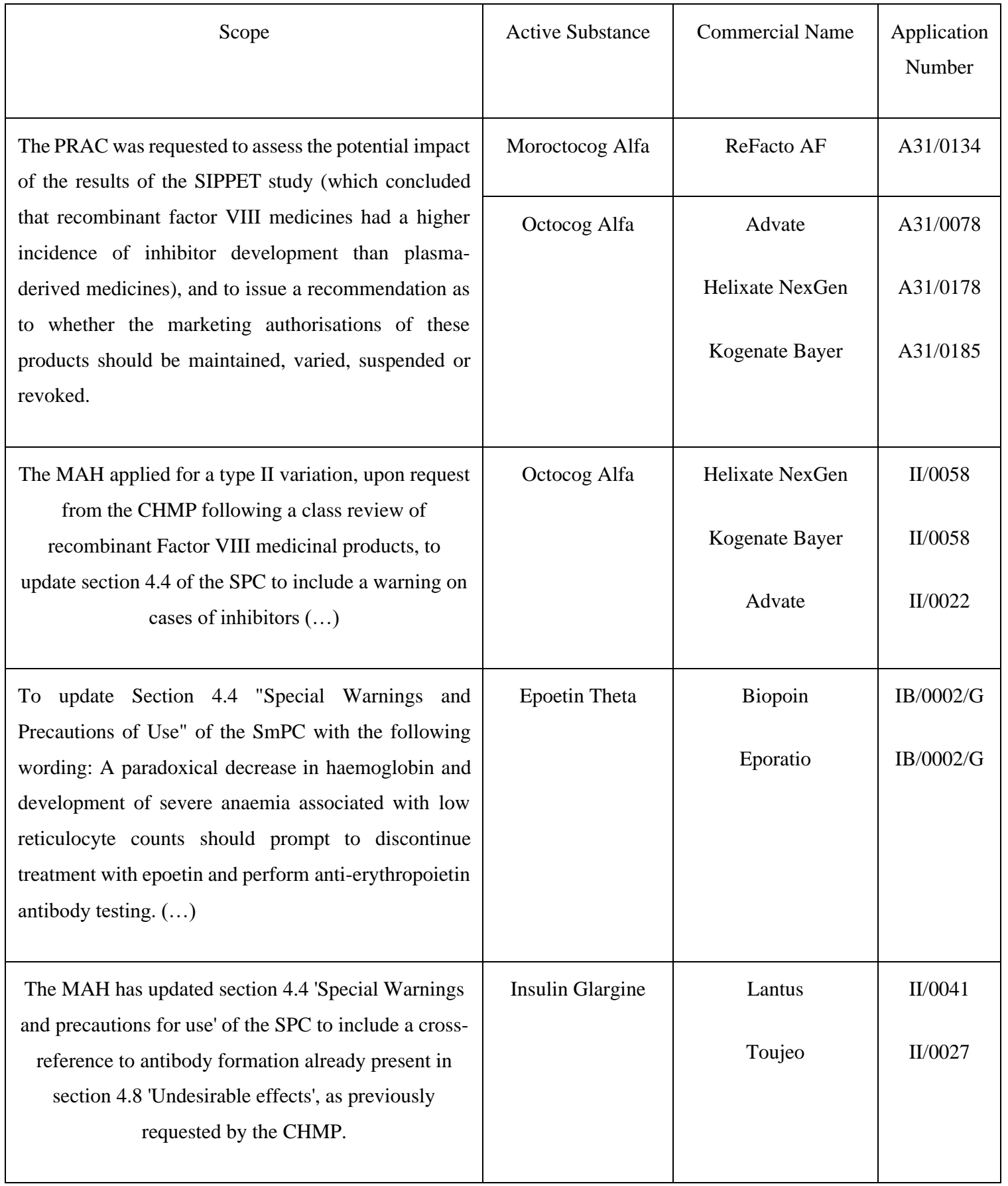

\section{References:}


1. Borrega R, Cruz JP, Taylor P, Goncalves J. Analysis of Immunogenicity data in the Product Information of Biological drugs: A need to report immunogenicity data systematically.

2. Pineda C, Hernández GC, Jacobs IA, Alvarez DF, Carini C. Assessing the Immunogenicity of Biopharmaceuticals. BioDrugs. 2016;30:195-206

3. European Medicines Agency. EMA/168402/2014- Guideline on good pharmacovigilance practices (GVP) Product- or Population-Specific Considerations II: Biological medicinal products. Available at: https://www.ema.europa.eu/en/documents/scientific-guideline/guideline-goodpharmacovigilance-practices-gvp-product-population-specific-considerationsii_en-0.pdf [Accessed March 2019]

4. European Biopharmaceutical Enterprises. Tell me the whole story: the role of product labelling in building user confidence in biosimilars in Europe. GABI Journal. 2014;3(4):188-92.

5. European Medicines Agency, European Commission. Biosimilars in the EU Information guide for healthcare professionals. Available at: https://www.ema.europa.eu/en/documents/leaflet/biosimilars-eu-informationguide-healthcare-professionals_en.pdf [Accessed April 2019]

6. Dolinar RO, Reilly MS. Biosimilars naming, label transparency and authority of choice - survey findings among European physicians. GABI Journal. 2014;3(2):58-62

7. Ebbers HC, Chamberlain P. Controversies in Establishing Biosimilarity: Extrapolation of Indications and Global Labeling Practices. BioDrugs. 2016;30(1):1-8.

8. Shankar G, Arkin S, Cocea L, Devanarayan V, Kirshner S, Kromminga A, et al. Assessment and Reporting of the Clinical Immunogenicity of Therapeutic Proteins and Peptides - Harmonized Terminology and Tactical Recommendations. AAPS J. 2014;16(4):658-73.

9. Gunn GR, Sealey DCF, Jamali F, Meibohm B, Ghosh S, Shankar G. From the bench to clinical practice: understanding the challenges and uncertainties in immunogenicity testing for biopharmaceuticals. Clin Exp Immunol. 2016;184(2):137-46.

10. Belmonte RG, Chirlaque CH, Amador MA, Aranda CJ, González R, Augustin OM, et al. Biosimilars: concepts and controversies. Pharmacol Res. 2018;133:251-64.

11. Cohen HP, Blauvelt A, Rifkin RM, Danese S, Gokhale SB, Woollett G. Switching Reference Medicines to Biosimilars : A Systematic Literature Review of Clinical Outcomes. Drugs. 2018;78(4):463-78.

12. Mckinnon RA, Cook M, Liauw W, Marabani M, Marschner IC, Packer NH, et al. Biosimilarity and Interchangeability: Principles and Evidence: A Systematic Review. BioDrugs. 2018;32(1):27-52.

13. European Medicines Agency. ReFacto AF- Procedural steps taken and scientific information after the authorisation. Available at: https://www.ema.europa.eu/en/documents/procedural-steps-after/refacto-afepar-procedural-steps-taken-scientific-information-after-authorisation_en.pdf [Accessed March 2019]

14. European Medicines Agency. Helixate NexGen- Procedural steps taken and scientific information after the authorisation. Available at: https://www.ema.europa.eu/en/documents/procedural-steps-after/helixatenexgen-epar-procedural-steps-taken-scientific-information-after- 
authorisation_en.pdf [Accessed March 2019]

15. European Medicines Agency. Biopoin- Procedural steps taken and scientific information after the authorisation. Available at:

https://www.ema.europa.eu/en/documents/procedural-steps-after/biopoin-eparprocedural-steps-taken-scientific-information-after-authorisation_en.pdf [Accessed March 2019]

16. European Medicines Agency. Eporatio- Procedural steps taken and scientific information after the authorisation. Available at:

https://www.ema.europa.eu/en/documents/procedural-steps-after/eporatio-eparprocedural-steps-taken-scientific-information-after-authorisation_en.pdf [Accessed March 2019] 